Originally published as:

Lankester, F., Mätz-Rensing, K., Kiyang, J., Jensen, S.A., Weiss, S., Leendertz, F.H. Fatal ulcerative colitis in a western lowland gorilla (gorilla gorilla gorilla) (2008) Journal of Medical Primatology, 37 (6), pp. 297-302.

DOI: 10.1111/j.1600-0684.2008.00287.x

The definitive version is available at: http://onlinelibrary.wiley.com/ 


\title{
Fatal ulcerative colitis in a western lowland gorilla (gorilla gorilla gorilla)
}

\author{
F. Lankester ${ }^{1}$, K. Mätz-Rensing ${ }^{2}$, J. Kiyang ${ }^{1}$, S.A. Jensen ${ }^{3}$, S. Weiss ${ }^{3}$ \& F.H. Leendertz ${ }^{3}$ \\ ${ }^{1}$ The Limbe Wildlife Centre, Limbe, Cameroon \\ ${ }^{2}$ German Primate Centre, Göttingen, Germany \\ ${ }^{3}$ Robert Koch-Institut, Berlin \& Max-Planck-Institute for Evolutionary Anthropology, Leipzig, Germany
}

\begin{abstract}
A captive western lowland gorilla (Gorilla gorilla gorilla) presented with watery diarrhoea that progressed to become profuse and haemorrhagic. Faecal analyses revealed Balantidium (B.) coli trophozoites and salmonella-like bacteria. Despite treatment the gorilla died on the 5th day after onset of symptoms. Post-mortem examination revealed a severe erosiveulcerative superficial and deep colitis. Histological examination of post-mortem samples of the colon showed plentiful B. coli invading into the mucosa and submucosa, whilst PCR screening of bacterial DNA could not confirm any bacteria species which could be connected to the clinical picture. As B. coli is usually a nonpathogenic gut commensal, and as this animal previously showed evidence of non-symptomatic infection of $\mathrm{B}$. coli, it is possible that the switch in pathogenicity was triggered by an acute bacterial infection. Despite successful treatment of the bacterial infection the secondary deep invasion of B. coli was not reversed, possibly because of the failure of the treatment regimen, and led to the death of the gorilla.
\end{abstract}

\section{Introduction}

Enteric diseases are considered the primary cause of morbidity and mortality in captive gorillas [Gorilla (G.) gorilla (g.)] with the bacteria shigella, salmonella and a variety of macro parasites, principally balantidia and strongyloides, being the most frequently isolated pathogens [3, 7, 14]. Balantidium (B.) coli, a large ciliated protozoan with worldwide distribution, inhabits the gastrointestinal tract of many mammalian species,s ome birds and arthropods, although the pig is considered to be the principal natural reservoir host $[9,18]$. In captive gorillas infections with B. coli are usually non-clinical. In some cases a mild, self-limiting disease may result on first exposure to the protozoa [18]. However, under certain conditions, such as concurrent infections with an agent, stress or poor sanitary conditions resulting in a high parasitic burden, B. coli can become invasive, penetrating deeply beyond the muscularis mucosa resulting in a severe clinical condition. The consequent lesions may also perforate the wall of the gastrointestinal tract, producing peritonitis. Balantidia may also penetrate the lymphatic system or blood vessels and spread to other organs, most commonly the liver. Invasive B. coli infection is often fatal in the gorilla $[9,18]$ particularly if the condition remains untreated.

This report describes the clinical picture, pathology and histology of a fatal case of invasive balantidiasis in an 11-year-old female captive western lowland gorilla (Gorilla gorilla gorilla), that was possibly triggered by infection with salmonella.

\section{Case report}

\section{Animal and housing}

An 11-year-old female western lowland gorilla weighing $80 \mathrm{~kg}$ shared an enclosure with 10 other western lowland gorillas and one Cross-River gorilla (G. g. diehli) at the Limbe Wildlife Centre (LWC), South West Province, Cameroon. The enclosure consisted of a $2500 \mathrm{~m} 2$ outdoor area, with natural grass-covered earth substrate, and a $750 \mathrm{~m} 3$ indoor 'night house', constructed from steel piping, with five interconnecting chambers, which was cleaned daily using $0.25 \%$ sodium hypoclorite bleach solution. The group were fed a diet consisting of locally harvested browse, seasonal fruit and vegetables, home-made natural yogurt and boiled chicken's eggs. The female gorilla had been living at the LWC for 22 months prior to this case, during which time she did not suffer from any periods of sickness, had been intra-dermally tested four times for tuberculosis (results negative), received 
anthelmintic treatment every 3 months, received a full vaccination course against tetanus and had been diagnosed as negative both for Simian T-lymphotropic Virus (STLV) and hepatitis B virus (surface antigen test).

\section{Clinical history \\ Day one}

The gorilla developed a markedly reduced appetite, watery green diarrhoea, was depressed and, on manual palpation during physical examination, showed signs of abdominal discomfort. All the other gorillas in the group were clinically normal. A clinical examination performed on the conscious gorilla indicated that she was neither pyrexic nor dehydrated and her mucous membranes were of normal colour. A direct examination of a fresh faecal sample under a light microscope revealed a moderate number (15 per slide preparation) of $\mathrm{B}$. coli trophozoites. The gorilla was immediately isolated from the group.

\section{Day two}

The gorilla was still depressed and had watery green diarrhoea. The faeces contained $17 \mathrm{~B}$. coli trophozoites per slide preparation, and in addition leucocytes were present (19 cells per slide preparation). Treatment was commenced consisting of $1000 \mathrm{mg}$ metronidazole (Flagyl®; Aventis, Paris, France) per os twice a day and $150 \mathrm{mg}$ marbofloxacin (Marbocyl囚10\%; Vétoquinol, Cedex, France) intramuscularly (IM) once daily. A fresh faecal sample was collected for culture and sensitivity testing at a local laboratory. After 48 hours of aerobic incubation at $37^{\circ} \mathrm{C}$ in Salmonella and Shigella Selective Agar (Pronadisa Microbiological Culture Media and Diagnostic Reagent) (Conda ${ }^{\circledR}$, Madrid, Spain), a moderate bacterial growth was identified in the laboratory, based on the colour and odour, as being a salmonella species. Sensitivity tests revealed that the colonies were sensitive to norfloxacin, ofloxacin, perfloxacin and gentamycin.

\section{Day three}

The gorilla's condition had not improved; she was depressed, recumbent and her appetite remained reduced, although she drank water from the tap in her cage as well as oral rehydration fluids (ORF) (Pediatric Electrolyte; Nutramax Products, Inc. Gloucester, MA, USA) that were offered to her. Her rectal temperature was $38^{\circ} \mathrm{C}$, her mucous membranes were pale pink, and, on palpation during physical examination, showed signs of abdominal pain. Her hydration status was assessed as being normal. The number of B. coli trophozoites in the faeces had increased to more than 100 per preparation. At this stage, the gorilla began refusing to take medication orally and so, to facilitate

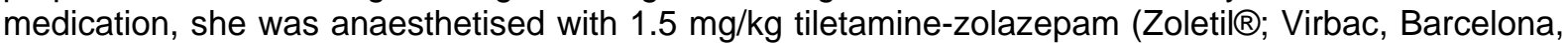
Spain) and $0.015 \mathrm{mg} / \mathrm{kg}$ medetomidine hydrochloride (Domitor ${ }^{\circledR}$; Animal Health, Pfizer Limited, Kent, UK) administered IM. The anaesthesia was reversed after 25 minutes with $0.075 \mathrm{mg} / \mathrm{kg}$ atipamezole hydrochloride (Antisedan $\AA$; Animal Health) administered IM. During the anaesthesia $1000 \mathrm{mg}$ metronidazole was administered per vaginal suppository and $150 \mathrm{mg}$ of marbofloxacin was administered IM. A white blood cell count, calculated using the Unopette ${ }^{\circledR}$ In Vitro Diagnostic System (Unopette ${ }^{\circledR}$; Becton Dickinson Vacutainer Systems, Franklin Lakes, NJ, USA) revealed a mild leucocytosis $(11.975 \cdot 109 / /)$ with a neutrophilia with a regenerative left shift. The packed cell volume was calculated at 36\%, using a Zipocrit Microhematocrit Centrifuge and Reader Card (LW Scientific, Inc., Tucker, GA, USA).

\section{Day four}

The gorilla continued to be depressed, although she was walking around her cage, eating small amounts and drinking water and ORF. The gorilla's temperature was $37.5^{\circ} \mathrm{C}$. In the morning, the diarrhoea was watery and green but by afternoon it had become haemorrhagic. Examination of a direct smear of the faeces revealed over $100 \mathrm{~B}$. coli trophozoites per slide preparation. To administer medication she was sedated in the morning and evening, with an IM injection of $1.5 \mathrm{mg} / \mathrm{kg}$ ketamine hydrochloride (Ketaset ${ }^{\circ}$ Injection; Fort Dodge Animal Health Ltd, Southampton, UK). Metronidazole and marbofloxacin were administered as per the previous day. Clinical examination revealed that she was dehydrated and the mucous membranes were pale, with a capillary refill time of $3 \mathrm{~s}$. Abdominal sounds were reduced. One litre of warmed Hartmann's Solution (Lactate-Ringer; Medicam, Société Industrielle des Produits Pharmaceutiques, Yaoundé , Cameroon) was administered rectally and 1 I subcutaneously during each sedation.

\section{Day five}

The gorilla's condition had significantly deteriorated. She was severely depressed and lethargic, with her eyes rolling back when left undisturbed. The haemorrhagic diarrhoea continued. However, she did eat small amounts of food, and was drinking voluntarily from the tap. In the morning and in the evening 
the gorilla was sedated with $1 \mathrm{mg} / \mathrm{kg}$ ketamine hydrochloride administered IM. Metronidazole, marbofloxacin and Hartmann's solution were administered as per the previous day. After waking from the second sedation she developed tachycardia, became increasingly weak, her mucous membranes became pale, and the rectal temperature dropped to $33.8^{\circ} \mathrm{C}$. Dexamethasone sodium phosphate $(0.5$ $\mathrm{mg} / \mathrm{kg}$ ) (Butler®; The Butler Company, Columbus, OH, USA) was administered IM; however, the gorilla did not respond to this treatment and died 3.5 hours later.

\section{Pathology and histology}

The primary gross pathological findings from the post-mortem examination were discovered in the large intestine: Extensive haemorrhage and bruising throughout the length of the colon, with circular ulcerative lesions, measuring approximately $0.5 \mathrm{~cm}$ in diameter, found on the mucosal surface of the ascending colon. Multiple pus-filled abscesses of up to $1 \mathrm{~cm}$ in diameter were present throughout the transverse and descending colon. The mesenteric lymph nodes were enlarged and haemorrhagic. The liver, weighing $2.25 \mathrm{~kg}$, was swollen and mustard in colour. Multiple tissue samples were collected and stored in 10\% formalin, RNAlater (Qiagen, Hilden, Germany), and 10\% glycerol and blood drops were collected and dried on filter paper according to Leendertz et al. [10].

Histological evaluation was performed on formalinfixed tissues that were embedded in paraffin wax at the German Primate Centre in Göttingen, Germany. Sections of 3-4 Im were stained routinely with haematoxylin-eosin stain (HE). Special stains including Giemsa stain, periodic acid-Schiff (PAS)reaction, Berlin Blue and Ziehl-Neelsen stain were used to evaluate the presence of infectious agents. The main histological alterations were found in the digestive system (Fig. 1). The colon showed a severe acute to sub-acute

erosive-ulcerative colitis, with some ulcers extending into deep parts of the submucosa, producing a predominantly lympho-histiocytic inflammatory infiltration in mucosal and submucosal parts of the intestinal wall. In the submucosa as well as in the whole ulcer area there was evidence of large numbers of B. coli trophozoites, which invaded deep into the submucosa. The mucous membranes showed superficial autolysis as well as necrotising processes, and the crypts presented evidence of abscesses after B. coli invasion. It was not evident from the tissue sections if the tissue alterations were caused by B. coli as a primary infection, or if B. coli invaded the damaged mucosa as a secondary pathogen. Other non-ulcerated parts of the intestine showed evidence of chronic enteritis characterised by hyperplasia of gut-associated lymphoid tissue and moderate lymphoplasmacellular inflammatory infiltration in the mucosa lamina.

\section{Molecular diagnostics}

DNA and RNA were extracted from colon and mesenteric lymph nodes preserved in RNAlater (Qiagen) using DNAeasy and RNAeasy tissue kits (Qiagen), according to the manufacturer's instructions. Screening for the presence of relevant gastrointestinal viruses was performed using PCRs targeting Enteroviruses, Noroviruses, Rotaviruses and Adenoviruses [12, 16] but all of these were negative. PCRs targeting the highly conserved $16 S$ rRNA region of the bacterial genome [11] were used to analyse the spectrum of bacteria present in these organs. PCR products were cloned using the Topo TA Cloning Kit (InvitrogenTM, Karlsruhe, Germany). Clones were selected and sequenced [17] on an ABI Prism 3100 Genetic Analyzer. Sequence information obtained from the bacteria screening was compared with sequences of the public databases for identification of the different species. Of the 27 sequences analysed, five showed homologies to Neisseria spp. and 22 homologies to sequences of Streptococcus thermophilus. S thermophilus is not a typical disease causing pathogen and can be considered a contamination through gut flora, environment or PCR. To determine whether the Neisseria sequences originated from the pathogenic Neisseria meningitides or from a non-pathogenic Neisseria, which could also have been a contamination, a specific PCR targeting N. meningitides [4] was performed and the samples tested negative.

\section{Discussion}

We have described a case of enteritis in a western lowland gorilla that began with watery green diarrhoea and progressed, with increasing faecal levels of B. coli trophozoites, to dysentery, collapse and death. Histological analyses of the colon showed that B. coli invaded tissue beyond the muscularis mucosa and produced deep ulcerations and abscesses. The trigger for the parasite's sudden increase in pathogenicity wasprobably because of a bacterial or a viral infection, in this case possibly salmonella, which is known to facilitate invasion of the parasite [18]. However, salmonella could not be detected using molecular methods on post-mortem samples. This is most likely a result of 
the successful treatment with antibiotics in the course of the disease. Faeces samples were not collected for molecular analysis before antibiotic treatment as the severity of the case was not obvious at that time.

All animals of the LWC gorilla group had been asymptomatic carriers of B. coli for a long period prior to the described case as trophozoites were often seen in their stool samples. Consequently, drugs against this parasite are not typically given on finding B. coli trophozoites in stool samples, unless faecal trophozoite counts rise sharply and/or clinical signs indicate that extraintestinal invasion may occur.

There are several drugs - metronidazole, tetracycline, paromomycin and chloroquine - reported to have been used, with varying degrees of efficacy, for the treatment of balantidiasis [9]. Of these drugs only metronidazole and tetracycline were locally available of which metronidazole was selected. Metronidazole had been used in previous cases at LWC in an attempt to eliminate B. coli from the stool of gorillas and chimpanzees (Pan troglodytes), with varying degrees of success, and with at least 10 days of treatment being required before a significant reduction in the number of trophozoites would be seen in fresh stool samples. Indeed, in several cases, trophozoite reduction was not seen even after 10 days of oral treatment.

The dosage of metronidazole that was aimed for was $10 \mathrm{mg} / \mathrm{kg}$ per os thrice a day for 7-10 days duration [13], however, in this particular case the gorilla only accepted two oral doses of $1000 \mathrm{mg}$ of metronidazole on day 1 and thereafter refused to drink any medicated drink. Consequently, intravaginal administration of metronidazole was subsequently attempted. The efficacy of administering metronidazole via the vagina is, however, uncertain. One study [5] indicated that metronidazole is rapidly absorbed through the vaginal mucosa of the rat, whereas another study [6] indicated that serum concentrations of metronidazole in humans after intravaginal administration were significantly less than the concentrations seen with the standard oral dose. Consequently, it is uncertain whether administration via the vagina can lead to therapeutic levels of the drug in the serum.

The effect of multiple sedations on the outcome of this case is difficult to quantify, as anaesthesia in general has negative effects on the body systems and adds physical stress to the animal. In addition, ketamine hydrochloride, a rapid acting dissociative anaesthetic, does affect the cardiovascular system by increasing cardiac output, heart rate and arterial and venous blood pressure [15]. Because of these hypertensive effects ketamine hydrochloride has a relative contra-indication in cases of significant blood loss. Therefore, it is possible that the repeated use of ketamine may have resulted in increased haemorrhage from the colon.

Administering fluid therapy in gorillas is difficult. Fluid restoration was first attempted via subcutaneous and rectal routes during the twice-daily sedations, but this was not sufficient to reverse the ongoing and increasing circulatory shock because of the severity of the haemorrhagic diarrhoea. Maintaining an intravenous line in the gorilla for long enough to allow sufficient fluids to be given for volume expansion and rehydration was neither possible because of the lack of substantial quantities of sedatives required nor wanted because of the negative impact of prolonged anaesthesia. The resulting dehydration most likely contributed to the death of the gorilla.

Following this case, another gorilla with similar symptoms was promptly treated with $250 \mathrm{mg}$ paramomycin sulphate (Caraco Pharmaceutical Laboratories, Ltd, Detroit, MI, USA) as an alternative therapeutic agent in the treatment of balantidiasis [9]. The drug was administered per os twice a day for 5 days and, 24 hours after the start of treatment, the number of trophozoites of B. coli found per slide preparation of a direct faecal smear had decreased to zero and the gorilla subsequently made a full recovery within 9 days. This rapid response to paromomycin therapy has been observed in gorillas and chimpanzees at the LWC on several occasions.

The primary infection that triggered the secondary balantidiasis invasion of the gastrointestinal wall was possibly salmonella. However, the faecal culture was performed in a local laboratory and the diagnosis was based on the typical odour and colour of the colonies grown in the salmonella and shigella selective media. Without the capability to perform further bacteriological tests, these findings are presumptive.

The decision to use antimicrobials for the treatment of suspected cases of salmonellosis depends on their severity and the likelihood of an extra-intestinal spread of the infection [2, 8]. In mild-to-moderate cases, which are usually self-limiting, the use of antibiotics is not recommended as their use can 
prolong the excretion of the organism. Instead, symptomatic treatments such as a bland diet, electrolytes and fluids typically suffice [2]. However, in severe cases, where any risk factors for developing extraintestinal spread are present, antimicrobial therapy with fluoroquinolones, trimethoprim-sulfamethoxazole, ampicillin, or a thirdgeneration cephalosporin should be initiated $[2,8]$. In this particular case, the deterioration in clinical signs and the appearance of leucocytes in the stool sample on day 2 suggested that an invasive process (whether driven by salmonella or B. coli or both) could be occurring. As such a decision to aggressively treat the condition with the fluoroquinolone marbofloxacin was taken. The antibacterial therapy is presumed to have been successful as no traces of any common pathogenic gut bacteria could be found on PCR analysis of the tissues collected at the time of the post-mortem examination. However, despite the fact that treatment of the primary infection was successful, it is presumed that the infection had already triggered a rapid increase in $\mathrm{B}$. coli trophozoites which inevitably led to the overwhelming invasion of the trophozoites deep into the submucosa of the colon.

The actual source of the presumed salmonella infection in this case remains uncertain. Of all salmonella infections in humans, approximately $80 \%$ originate from the ingestion of animal products (poultry, eggs and meats), or from animal vectors (flies, birds, lizards and rodents) [1, 8]. Animal products, such as boiled eggs and yogurt, are included in the diet of the LWC gorillas and the above animal vectors are all commonly found in the gorilla enclosure at the LWC and have to be considered as possible sources.

\section{Conclusion}

We have described a lethal case of severe ulcerative colitis because of $B$. coli in a captive lowland gorilla and illustrated how this protozoan can, once triggered by a concurrent infection such as salmonella, change from a gut commensal to a highly invasive fatal parasite. This report highlights the need for constant monitoring of parasite levels, the need for rapid and appropriate treatment of $B$. coli and other diseases that could possibly facilitate invasion of the parasite, as well as the difficulties that the administration of drugs and sufficient fluid therapy in a gorilla present.

\section{Acknowledgments}

We would like to thank Dr E. Schreier and his team from FG 15 Robert Koch-Institute for conducting the analyses for the gastrointestinal viruses and U. Buwit and J. Tesch for performing the sequence reactions. The work was carried out under the umbrella of the 'Great Ape Health Monitoring Unit', a network supported by the Robert Koch-Institute, Berlin, Germany, the Max-Planck-Institute for Evolutionary Anthropology, eipzig, Germany, the Woodland Park Zoo, Seattle, USA and the Cleveland, Metroparks Zoo, OH, USA.

\section{References}

1 Barro N, Aly S, Tidiane OCA, Sababénédjo TA: Carriage of bacteria by proboscisis, legs and faeces of two species of flies in food vending sites in Ouagadougou, Burkina Faso. J Food Prot 2006; 69:20070-100.

2 Beers MH, Berkow R: The Merck Manual of Diagnostic Therapy. Whitehouse Station, NJ: Merck Research Laboratories, 1999.

3 Benirschke K, Adams FD: Gorilla diseases and causes of death. J Reprod Fertil Suppl 1980; 28:139-48.

4 Bronska E, Kalmusova J, Dzupova O, Maresova V, Kriz P, Benes J: Dynamics of PCR-based diagnosis in patients with invasive meningococcal disease. Clin Microbiol Infect 2006; 12:137-41.

5 Butta HS, Siddiqu WH: Pharmacokinetics and metabolic disposition of 14C-metronidazole-derived radioactivity in rat after intravenous and intravaginal administration. Arch Int Pharmacodyn Ther 1980; 245: 4-19.

6 Cunningham FE, Kraus DM, Brubaker L, Fischer JH: Pharmacokinetics of intravaginal metronidazole gel. J Clin Pharmacol 1994; 34:1060-5.

7 Fox JG, Newcomer CE, Rozmiarek H: Selected zoonosis and other health hazards. In: Laboratory Animal Medicine. Fox, Cohen \& Loew (eds). Orlando, FL: Academic Press, 1984; 628-30. 
8 Kenji I, Kazuo K, Michio O: Bacterial haemorrhagic enterocolitis. J Gastroenterology 2003; 38:11120.

9 Lee RV, Prowten AW, Anthone S, Satchidanand SK, Fisher JE, Anthone R: Typhlitis due to Balantidium coli in captive lowland gorillas. Rev Infect Dis 1990; 12:1052-9.

10 Leendertz FH, Pauli G, Ellerbrok H, Mätz-Rensing K, Boardman W, Jensen SA, Junglen S, Boesch C: Pathogens as drivers of population declines: the importance of systematic monitoring in great apes and other threatened mammals. Biol Conserv 2006; 131:325-37.

11 Muyzer G, de Waal EC, Uitterlinden AG: Profiling of complex microbial populations by denaturing gradient gel electrophoresis analysis of polymerase chain reaction-amplified genes coding for $16 \mathrm{~S}$ rRNA. Appl Environ Microbiol 1993; 59:695-700.

12 Oh DY, Gaedicke G, Schreier E: Viral agents of acute gastroenteritis in German children: prevalence and molecular diversity. J Med Virol 2003; 71:82-93.

13 Pan African Sanctuaries Alliance. Primate Veterinary Healthcare Manual, 1st edn. Pan African Sanctuaries Alliance, Portland, Oregon, 2004; 64.

14 Paul-Murphy J: Bacterial enterocolitis in non-human primates. In: Zoo and Wild Animal Medicine Current Therapy, Vol. 3. Fowler (ed.), Philadelphia: W. B. Saunders, 1993; 544.

15 Plumb DC: Plumb's Veterinary Drug Handbook, $5^{\text {th }}$ edn. Wisconsin: PharmaVet Inc., 2005; 439-40. 16 Pusch D, Oh DY, Wolf S, Dumke R, Schröter-Bobsin U, Höhne M, Röske I, Schreier E: Detection of enteric viruses and bacterial indicators in German environmental waters. Arch Virol 2005; 150:929-47. 17 Sanger F, Nicklen S, Coulson AR: DNA sequencing with chain-terminating inhibitors. Proc Natl Acad Sci USA 1977; 74:5463-7.

18 Teare JA, Loomis MR: Epizootic of balantidiasis in lowland gorillas. J Am Vet Med Assoc 1982; 181:1345-7.

\section{Figures}
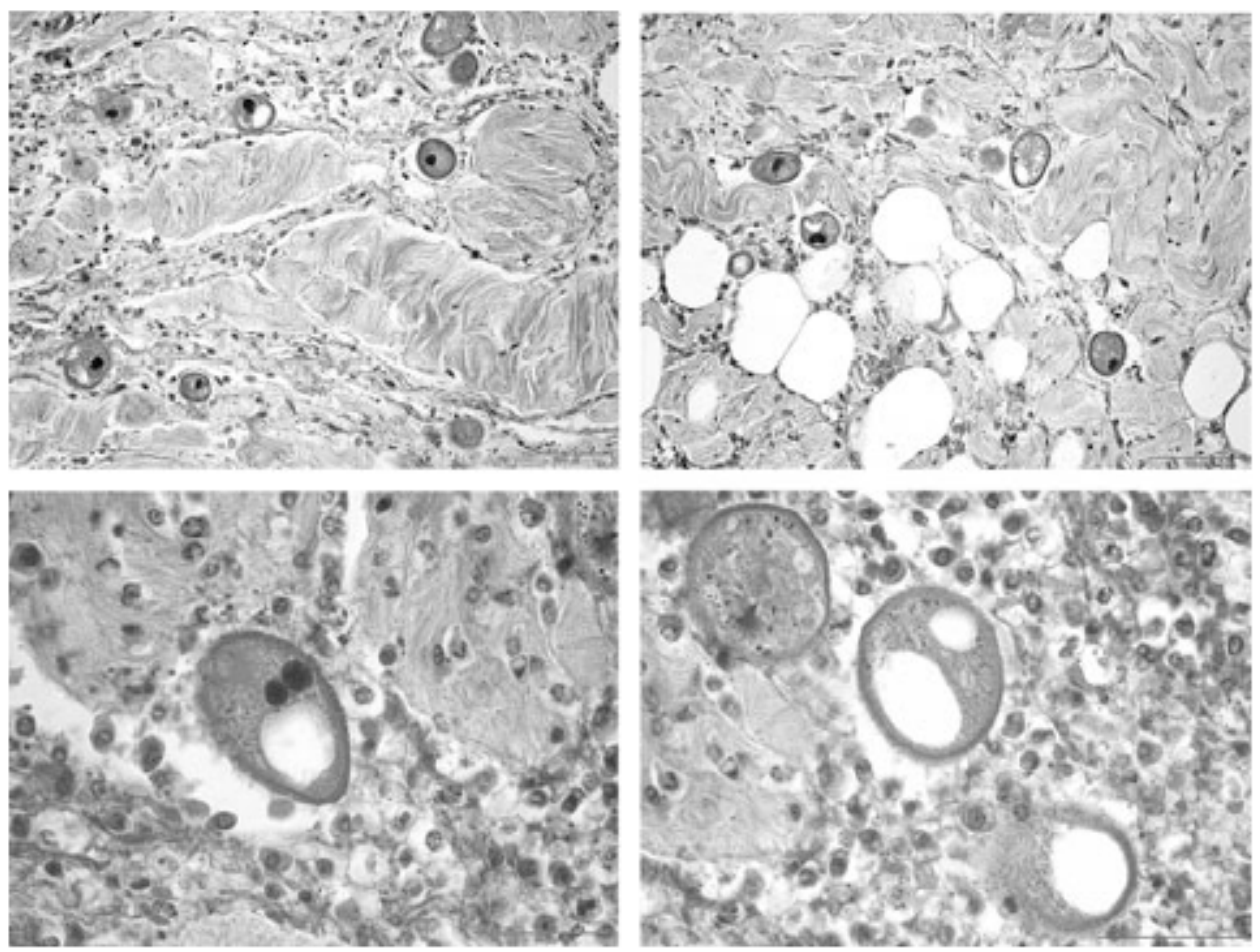

Figure 1 Photomicrograph of a colon section from the gorilla infected with Balantidium coli invading into deep parts of the submucosa. The throphozoites are egg-shaped with a pointed posterior end. HE-stain. 